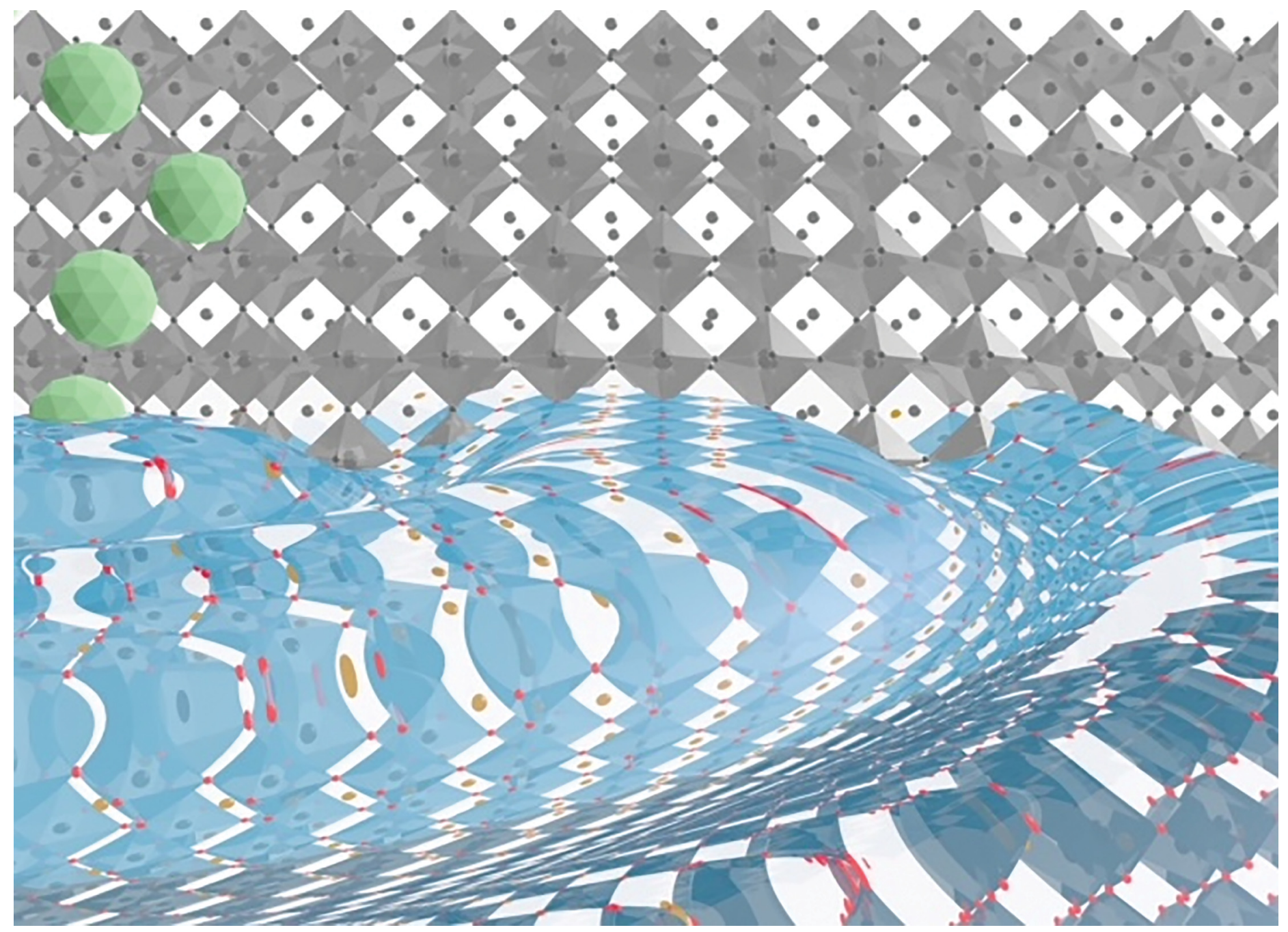

Showcasing research from Professor Trudel's laboratory, Department of Chemistry, University of Calgary, Alberta, Canada.

Water-dispersible and ferroelectric PEGylated barium titanate nanoparticles

Many applications require functional nanomaterials to be dispersed in aqueous media, which for some materials still proves challenging. One such example is barium titanate, the prototypical ferroelectric material. In this work, barium titanate nanoparticles that are dispersible in water are synthesized in a simple, one-step synthesis by coating them with poly (ethylene glycol). These nanoparticles retain their ferroelectricity at the nanoscale. This development opens up avenues ranging from electrorheological fluids to biomedical imaging.

\section{As featured in:}

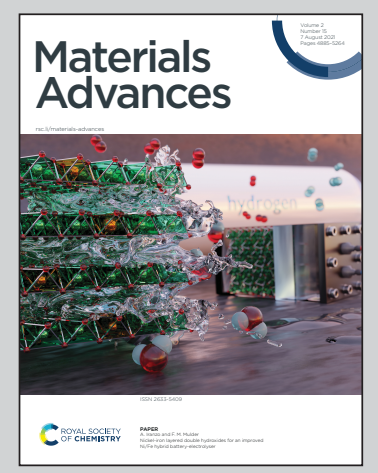

See S. Trudel et al.,

Mater. Adv., 2021, 2, 5089. 
Check for updates

Cite this: Mater. Adv., 2021, 2, 5089

Received 8th April 2021,

Accepted 13th June 2021

DOI: 10.1039/d1ma00317h

rsc.li/materials-advances

\title{
Water-dispersible and ferroelectric PEGylated barium titanate nanoparticles $\dagger$
}

\author{
M. Taheri, ${ }^{a b c}$ B. Zanca, ${ }^{a}$ M. Dolgos, (D) ${ }^{a}$ S. Bryant ${ }^{c}$ and S. Trudel (D) ab $^{a b}$
}

\begin{abstract}
Dispersions of ferroelectric nanoparticles in aqueous medium can find promising applications in electrooptical, medical, and smart fluid technologies. In this report, we show the development of highly dispersed nano-sized ferroelectric barium titanate $\left(\mathrm{BaTiO}_{3}\right)$ powders with high dielectric constant prepared using a simple, one-step low temperature solution method. The surface of these tetragonalstructured nanoparticles were modified with poly(ethylene glycol) as a stabilizer and dispersant. The crystal structure, morphology and dielectric constant of samples are discussed in detail. The colloidal stability and surface behavior of these PEGylated barium titanate nanoparticles are studied by means of $\zeta$-potential and dynamic light scattering measurements. We show changing the reaction conditions allows to tune the nanoparticle size. This research promotes a pathway to develop advanced ferroelectric nanomaterials with engineered properties in a simple way.
\end{abstract}

\section{Introduction}

Perovskite-type barium titanate $\left(\mathrm{BaTiO}_{3}\right)$ with four temperaturedependent crystalline forms (i.e. cubic, tetragonal, orthorhombic, and rhombohedral) is the most widely used ferroelectric (FE) material with applications in the electrical and electronic industries, mainly as multilayer ceramic capacitors, piezoelectric sensors and dielectrics. ${ }^{1}$ The tetragonal phase (space group $P 4 / \mathrm{mmm}$, see Fig. S1, ESI $\dagger$ ) supports a permanent electric polarization and a high dielectric constant $\left(\varepsilon_{\mathrm{r}}^{\prime} \approx 5000-15000\right)$ up to the FE Curie-Weiss temperature $\left(\approx 130{ }^{\circ} \mathrm{C}\right)$; at higher temperatures it converts to a fully symmetric cubic structure and becomes paraelectric. In this symmetric phase, $\mathrm{BaTiO}_{3}$ shows a temporary polarization under an application of electric field. It is well known that the tetragonal phase of $\mathrm{BaTiO}_{3}$ switches to the cubic non-ferroelectric phase at room temperature below a critical size. ${ }^{2}$ Reported critical sizes vary from as small as a few $\mathrm{nm}^{3,4}$ to $c a .100 \mathrm{~nm}^{5}$

Stable FE nanoparticle (NP) suspensions are required for various proposed applications including drug delivery, imaging, solution-based ceramics and coatings fabrication, and pigments. ${ }^{6-8}$ Recent efforts have focused on the dispersion of

\footnotetext{
${ }^{a}$ Dept. of Chemistry, University of Calgary, 2500 University Drive NW, Calgary, $A B$, Canada.E-mail:trudels@ucalgary.ca,maryam.taheri2@ucalgary.ca

${ }^{b}$ Institute for Quantum Science and Technology, University of Calgary, 2500 University Drive NW, Calgary, $A B$, Canada

${ }^{c}$ Dept. of Chemical and Petroleum Engineering, University of Calgary,

2500 University Drive NW, Calgary, AB, Canada

$\dagger$ Electronic supplementary information (ESI) available: Synthetic details, X-ray diffraction results, scanning electron micrographs, HRTEM of sample BT-1. See DOI: $10.1039 / \mathrm{d} 1 \mathrm{ma} 00317 \mathrm{~h}$
}

ferroelectric $\mathrm{BaTiO}_{3}$ nanopowders in either aqueous or organic media. Polymer surface stabilization is an approach conducive to this goal. Various polymers or polyelectrolytes, including ammonium salts of poly(acrylic acid) (PAA), ${ }^{6}$ poly-L-lysine, ${ }^{8}$ and poly(vinylpyrrolidone $)^{9,10}$ have been used. Pang et al. developed amphiphilic $\mathrm{BaTiO}_{3}$ NPs using unimolecular star-like poly(acrylic acid)-block-polystyrene diblock copolymers. ${ }^{11}$ Similarly, Jiang et al. synthesized amphiphilic poly(vinylidene fluoride)(PVDF)- $\mathrm{BaTiO}_{3}$ nanocomposites using a PAA-blockPVDF building block, where the PAA block was used a scaffold for $\mathrm{BaTiO}_{3}$ growth, yielding tunable nanoparticle sizes and PVDF coating thickness. ${ }^{12}$

Since these ceramic particles boast superior ferroelectric/ piezoelectric/pyroelectric properties, are competitively low-cost, non-toxic and bio-compatible, they present great potential for use in electrorheological fluids and biomedical imaging applications. ${ }^{8,13}$ Several synthesis methods for the preparation of $\mathrm{BaTiO}_{3}$ nanoparticles have been proposed, including high temperature solid-state reaction, ${ }^{14}$ sol-gel, ${ }^{15}$ co-precipitation, ${ }^{16}$ and hydrothermal ${ }^{17}$ approaches. However, most of these methods are not conducive to making uniform well-dispersed $\mathrm{BaTiO}_{3}$ nanoparticles. Bare $\mathrm{BaTiO}_{3}$ particles are not thermodynamically stable in water/organic solvents, specifically in acidic aqueous dispersions where $\mathrm{Ba}^{2+}$ ions are leached out from the surface of $\mathrm{BaTiO}_{3}$ molecules. Therefore adding surfactants, polymers or polyelectrolytes is needed to make them dispersible in the liquid phase. $^{18}$

We chose poly(ethylene glycol) (PEG) to stabilize aqueousdispersed $\mathrm{BaTiO}_{3}$ nanoparticles based on its high solubility in water, bio-compatibility and eco-friendliness, and its welldocumented ability to solubilize metal and metal oxide 
nanoparticles. ${ }^{19-22}$ Direct synthesis in PEG through a one-pot synthesis makes upscaling of $\mathrm{BaTiO}_{3}$ production feasible. ${ }^{20}$

Although PEG has been widely used in the synthesis of nanoparticles to promote stability control and nanoparticle size, ${ }^{22}$ its use to disperse $\mathrm{BaTiO}_{3}$ nanoparticles is limited. ${ }^{23-25}$ Moreover, modification with PEG is suggested to improve the dielectric properties of ceramic particles. ${ }^{2,23,26}$ In addition to improving NP stability in solution, PEG-modified metal oxides were used to form high-quality ferroelectric thin films such as strontium- ${ }^{27}$ and lead-doped ${ }^{28} \mathrm{BaTiO}_{3}$.

In this work, we present the preparation and characterization of PEGylated $\mathrm{BaTiO}_{3}$ nanoparticles prepared using a lowtemperature one-pot synthesis. We synthesise tetragonal $\mathrm{BaTiO}_{3}$-PEG core-shell nanoparticles in the 40-70 $\mathrm{nm}$ diameter range, with high water dispersibility and large dielectric constant. We show how we can modify the synthetic parameters to control the particle size, and their corresponding stability in water. Furthermore, we report the temperature-dependence dielectric constant of a selected sample. This method is fast, simple, and cost-effective, attributes that are necessary for largescale industrial deployment.

\section{Experimental methods}

\subsection{Nanoparticle synthesis}

PEGylated $\mathrm{BaTiO}_{3}$ nanoparticles were prepared using a onestep protocol. All reactions were carried out under stirring and inert nitrogen atmosphere while the temperature was monitored and controlled with a digital PID controller. All syntheses are fully detailed in the ESI. $\dagger$ In a representative synthesis to prepare sample BT-1, we mixed powdered $\mathrm{Ba}(\text { acac })_{2} \cdot \mathrm{H}_{2} \mathrm{O}$ (355 mg, $1 \mathrm{mmol})$ and $(\mathrm{O}-\mathrm{i}-\mathrm{Pr})_{2} \mathrm{Ti}(\mathrm{acac})_{2}$ solution $(273 \mathrm{mg}, 1 \mathrm{mmol})$ in $3 \mathrm{~mL}$ of $\mathrm{PEG}_{400}$ in a round-bottom flask under nitrogen atmosphere, and let the solution stir for $30 \mathrm{~min}$. We then added aqueous $\mathrm{KOH}(6 \mathrm{~mL}, 1.5 \mathrm{M})$ to the mixture to adjust the $\mathrm{pH}$ of the solution to $\mathrm{ca}$. 14, which was found to be crucial for the nucleation of $\mathrm{BaTiO}_{3}$ particles. ${ }^{29}$ Immediately after, we increased the reaction temperature to reflux ( $c a .100 \pm 5{ }^{\circ} \mathrm{C}$ ), and allowed the reaction to reflux for $2 \mathrm{~h}$. The color of the solution gradually changed from orange/brown to white. After $2 \mathrm{~h}$ of reflux, we added $6 \mathrm{~mL}$ of distilled water to the mixture and maintained it at $100 \pm 5{ }^{\circ} \mathrm{C}$ for an additional $2 \mathrm{~h}$. We then opened the system to air and let the reaction cool down to room temperature. White precipitates were collected by washing and centrifugation (6000 rpm for $10 \mathrm{~min}$ ) two times with ethanol, followed by formic acid $(1 \mathrm{M})$. Carbonate impurities were removed by washing the product with diluted $(0.5 \mathrm{w} / \mathrm{w} \%)$ acetic acid. Finally, the nanoparticles were dried at $60{ }^{\circ} \mathrm{C}$ in a vacuum oven overnight.

In order to study the effect of reaction conditions on particle size and dispersibility, we modified the ratio of the metal precursors ( $\mathrm{Ti}$ and $\mathrm{Ba}$ molar ratio), $\mathrm{KOH}$ and PEG for samples BT-1 to BT-5 as summarized in Table 1. We prepared sample BT-0 under similar conditions to BT-1, without adding PEG, to provide a reference sample with no surface modification.
Table 1 Reaction conditions and impact on $\mathrm{BaTiO}_{3}$ nanoparticle sizes determined from XRD, SEM, and TEM data

\begin{tabular}{lllllll}
\hline Sample & $\begin{array}{l}{[\mathrm{Ba}]} \\
{[\mathrm{Ti}]}\end{array}$ & $\begin{array}{l}\text { relative } \\
{[\mathrm{PEG}]}\end{array}$ & $\begin{array}{l}{[\mathrm{KOH}]} \\
(\mathrm{M})\end{array}$ & $\begin{array}{l}D_{\text {XRD }} \\
(\mathrm{nm})\end{array}$ & $\begin{array}{l}D_{\text {SEM }} \\
(\mathrm{nm})\end{array}$ & $D_{\text {TEM }}(\mathrm{nm})$ \\
\hline BT-1 & 1 & 1 & 1.5 & $39.8 \pm 2.1$ & $69.9 \pm 9.5$ & $57.6 \pm 10.3$ \\
BT-2 & 2 & 1 & 1.5 & $32.3 \pm 1.6$ & $47.9 \pm 7.7$ & $40.4 \pm 7.9$ \\
BT-3 & 1 & 2 & 1.5 & $31.2 \pm 1.8$ & $43.9 \pm 6.3$ & $40.2 \pm 5.4$ \\
BT-4 & 1 & 1 & 1.6 & $33.6 \pm 2.2$ & $39.8 \pm 6.4$ & $41.8 \pm 6.3$ \\
BT-5 & 1 & 1 & 1.7 & $29.9 \pm 2.4$ & $43.4 \pm 7.2$ & $37.4 \pm 4.8$
\end{tabular}

\subsection{Physical characterization}

Powder X-ray diffraction (PXRD). Structural characterization was carried out using a Bruker D8 ECO Advance powder diffractometer $(\mathrm{Cu} \mathrm{K}, \lambda=1.5406 \AA$, $25 \mathrm{~mA} \times 40 \mathrm{kV}$ power, in the range of $2 \theta=15-75^{\circ}$, and increment of $0.02^{\circ}$ ). Profile refinement of XRD patterns was performed with the Fullprof software. ${ }^{30}$ Crystallite size $\left(D_{\mathrm{XRD}}\right)$ was estimated using the Scherrer equation (see ESI $\dagger$ ). ${ }^{31}$

Raman spectroscopy. Raman spectra were collected using a WITec confocal Raman microscope (Alpha 300R WITec GmbH, Germany) in the range of 100 to $900 \mathrm{~cm}^{-1}$ using a $5.5 \mathrm{~mW}$ laser with a wavelength of $532 \mathrm{~nm}$.

Electron microscopy. A FEI Quanta 250 FEG field-emission scanning electron microscope (SEM), $120 \mathrm{kV}$ Hitachi $\mathrm{H7650}$ transmission electron microscope (TEM) and $200 \mathrm{keV}$ FEI Tecnai Osiris S/TEM were used to image the nanoparticles. Powders were mounted on SEM sample stubs using doublesided carbon adhesive tape. A drop of particles dispersed in ethanol was allowed to dry on a TEM carbon-coated copper grid. Micrographs were analysed using the ImageJ software. ${ }^{32}$

Fourier-transform infrared (FTIR) spectroscopy. FTIR spectra were recorded with an Agilent Cary 630 spectrum with a diamond attenuated total reflectance (ATR) probe, and reported as transmittance.

Dynamic light scattering (DLS) and $\zeta$ potentials. DLS data and $\zeta$-potential of dispersed $\mathrm{BaTiO}_{3}$ nanoparticles were analysed using a Malvern Zetasizer Nano ZS DLS system. For each timepoint, three sequential measurements were made at room temperature to estimate the size, surface charge and dispersiblity of 100 ppm dried $\mathrm{BaTiO}_{3}$-PEG nanoparticles dispersed in DI water (1 mg nanoparticles in $10 \mathrm{~mL}$ DI water).

Ferroelectric properties. In order to study the ferroelectricity of sample BT-1, a pellet was fabricated by mixing powder with a solution of $3 \mathrm{wt} \%$ poly(vinyl butyral) in ethanol in a ratio of 10 drops per gram. The mixture was pressed into the cylindrical pellet using a Carver uniaxial press and a 13-mm stainless steel die. Three metric tons of pressure was applied and held for 2 min to ensure the integrity of the pellet. Recently, we studied the variation of grain size through the conventional one-step and two-step sintering techniques for micron- and nano-sized particle and concluded that two-step sintering can achieve high relative densities, while minimizing grain growth. ${ }^{33}$ Here, the BT-1 pellet was sintered in an alumina boat (CoorsTek) using a two-step temperature profile. The pellet was first heated to $1300{ }^{\circ} \mathrm{C}$ at a rate of $5{ }^{\circ} \mathrm{C} \mathrm{min}^{-1}$, held at that temperature for $1 \mathrm{~min}$, then cooled to $950{ }^{\circ} \mathrm{C}$ at a rate of $30{ }^{\circ} \mathrm{C} \mathrm{min}^{-1}$; this 
temperature was maintained for $10 \mathrm{~h}$ and the pellet was then cooled to room temperature at a rate of $5{ }^{\circ} \mathrm{C} \mathrm{min}^{-1}$.

The density $\rho_{\exp }$ of the pellet was measured with a Mettler Toledo analytical balance equipped with an Archimedes kit (with the accuracy of $\pm 0.01 \mathrm{~g} \mathrm{~cm}^{-3}$ ). The relative density $\rho_{r}$ of the pellet was calculated as $\rho_{\mathrm{r}}=100 \%\left(\rho_{\text {exp }} \cdot \rho_{\text {theo }}{ }^{-1}\right)$, where $\rho_{\text {theo }}$ is the theoretical crystallographic density obtained from XRD pattern refinements. To perform dielectric constant measurement, the pellet was polished on all sides using 800, 1200, and 4000 grit silicon-carbide polishing films (Stuers). Then, silver electrodes were painted on both sides of the pellet using a silver paint (SPI Supplies) and sintered at $550{ }^{\circ} \mathrm{C}$ for $15 \mathrm{~min}$ with a heating/cooling ramp rate of $5{ }^{\circ} \mathrm{C} \mathrm{min}^{-1}$. The dielectric properties were measured with a Solartron 1260 and 1296 coupled with a Probostat hightemperature sample stage inserted into a vertical tube furnace (Carbolite-Gero, VTF). The sample was heated/cooled at a rate of $1{ }^{\circ} \mathrm{C} \min ^{-1}$ from room temperature $\left(\approx 20-22{ }^{\circ} \mathrm{C}\right)$ to $200{ }^{\circ} \mathrm{C}$. Data was collected on both the heating and cooling cycles, every $5{ }^{\circ} \mathrm{C}$ until near the transition temperature $\left(T_{\mathrm{C}} \approx 115\right)$, then every $1{ }^{\circ} \mathrm{C}$ until after $T_{\mathrm{C}}$, then every $10{ }^{\circ} \mathrm{C}$. The tested frequencies ranged from $1 \mathrm{~Hz}$ to $100 \mathrm{kHz}$ with 5 points per decade.

\section{Results and discussion}

\subsection{Structural characterization and size control}

PXRD of the collected powders prior to the washing step indicated the major product of the reaction is $\mathrm{BaTiO}_{3}$. However, the presence of undesired $\mathrm{BaCO}_{3}$ was detected (Fig. S2a, ESI $\dagger$ ). This impurity is easily removed by washing the powders with diluted acetic acid $(0.5 \% \mathrm{w} / \mathrm{w})$, which is known to dissolve $\mathrm{BaCO}_{3} \cdot{ }^{34}$ After washing, all PXRD patterns show a singlephase product consisting of only $\mathrm{BaTiO}_{3}$ (see Fig. S2b, ESI $\dagger$ ).

The size of the crystalline $\mathrm{BaTiO}_{3}$ cores $\left(D_{\mathrm{XRD}}\right)$ were estimated from Scherrer's formula ${ }^{31}$ through fitting of the (110) peak (see Fig. S3 and eqn (S1), (S2), ESI $\dagger$ ). A size of $\sim 40 \mathrm{~nm}$ is determined for BT-1; $D_{\mathrm{XRD}}$ decreases from this reference point through increasing concentration of $\mathrm{Ba}^{+2}$, PEG content, or $\mathrm{KOH}(\mathrm{aq})$ molarity (see Table 1).

Fig. 1 shows the room-temperature PXRD pattern of sample BT-1. The observation of two closely related (002) and (200) reflections near $2 \theta \approx 45^{\circ}$ suggests the formation of a tetragonal structure with the space group $P 4 / \mathrm{mmm}$ (Fig. 1, inset), whereas a single (200) peak would be observed for cubic $\mathrm{BaTiO}_{3}$ (space group: $P m 3 m$ ). Rietveld refinement of the XRD pattern gives lattice parameters of $a=b=4.03 \AA$ and $c=4.05 \AA$ for sample BT-1, which are slightly larger than the lattice parameters of bulk $\mathrm{BaTiO}_{3}(a=b=3.99 \AA$ and $c=4.02 \AA)$ and in good agreement with published values of 4.03 to $4.05 \AA$ in $\mathrm{BaTiO}_{3}$ nanoparticles. ${ }^{35,36}$ The $c / a$ ratio of 1.005 suggests the structure is moderately tetragonal, while strong tetragonality value is reported to be about $1.010 .^{37}$ Table 2 summarizes the refined lattice parameters and selected bond distances. The data shows that the lattice parameters of samples with smaller particle sizes slightly increase, indicating smaller tetragonality value. Similarly, internal bond distances increase with this lattice

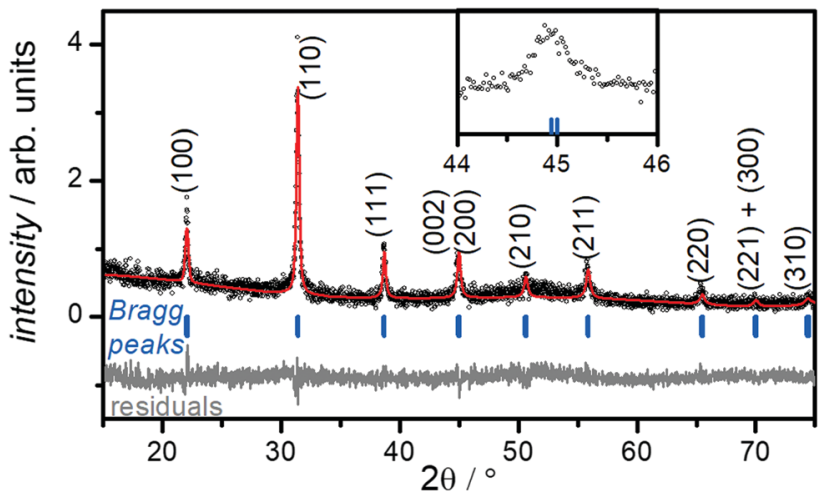

Fig. 1 Refined room temperature XRD pattern for BT-1. Scatter points are the measured data and are superimposed with the fit; the residual is plotted below. The position of the Bragg reflections is indicated by small vertical bars. The inset shows an small splitting of the (002) and (020) peaks, suggesting the formation of a moderately tetragonal structure.

expansion. Smaller nanoparticles can have larger unit cell, owing to various factors including defects, surface tension or symmetry reduction. A lattice expansion concurrent with size reduction has frequently been reported for $\mathrm{BaTiO}_{3}$ nanoparticles; this trend converts the tetragonal phase to the cubic phase structure in very small particles. ${ }^{38,39}$

It is well known that the presence of a tetragonal phase in $\mathrm{BaTiO}_{3}$ can be detected with Raman spectroscopy. ${ }^{40}$ To confirm the crystal structure of sample, we measured the Raman spectrum for sample BT-1, which is shown in Fig. S4 (ESI $\dagger$ ). Bands are observed at around 250 and $520 \mathrm{~cm}^{-1}$ (assigned to the transverse optical modes of A1 symmetry), around $720 \mathrm{~cm}^{-1}$, and a tetragonal characteristic peak at around $305 \mathrm{~cm}^{-1}$ (assigned to a B1 mode). ${ }^{40}$ The presence of the latter supports the tetragonal structure formation, in agreement with the XRD results presented above.

The morphology of PEGylated $\mathrm{BaTiO}_{3}$ nanoparticles was analyzed using SEM and TEM. As can be seen in the SEM micrographs (Fig. S5, ESI $\dagger$ ), BT-1 particles are uniformly quasispherical with rough surfaces ascribed to the PEG adsorbed on the nanoparticles' surfaces. The average particle size decreases from $\approx 70 \mathrm{~nm}$ for BT-1 to $\approx 40-48 \mathrm{~nm}$ in samples BT-2 to BT-5, with no change in the shape of the particles. The formation of $\mathrm{BaTiO}_{3}$-PEG core-shell structures in all samples was observed in the TEM micrographs (Fig. 2) with an average shell thickness of $c a$. 5-7 $\mathrm{nm}$ in the dry state. Lattice fringes can be observed in HRTEM images of BT-1 (Fig. 2b and Fig. S6, ESI $\dagger$ ), indicating the nanoparticles are single-crystal. The polymer layer is seen to

Table 2 Lattice parameters $(a, b, c)$ and internal atomic lengths, obtained from the refined XRD data

\begin{tabular}{lllllll}
\hline Sample & $a=b(\AA)$ & $c(\AA)$ & $c / a$ & $d_{\mathrm{Ba}-\mathrm{Ti}}(\AA)$ & $d_{\mathrm{Ti}-\mathrm{O} 1}(\AA)$ & $d_{\mathrm{Ti}-\mathrm{O} 2}(\AA)$ \\
\hline BT-1 & $4.03(1)$ & $4.05(2)$ & 1.005 & $3.49(8)$ & $2.02(6)$ & $2.01(6)$ \\
BT-2 & $4.05(7)$ & $4.07(5)$ & 1.004 & $3.51(9)$ & $2.03(8)$ & $2.02(9)$ \\
BT-3 & $4.04(9)$ & $4.06(4)$ & 1.004 & $3.51(1)$ & $2.03(2)$ & $2.02(5)$ \\
BT-4 & $4.04(5)$ & $4.06(4)$ & 1.004 & $3.50(8)$ & $2.03(2)$ & $2.02(3)$ \\
BT-5 & $4.04(6)$ & $4.06(5)$ & 1.004 & $3.50(9)$ & $2.03(3)$ & $2.02(2)$
\end{tabular}



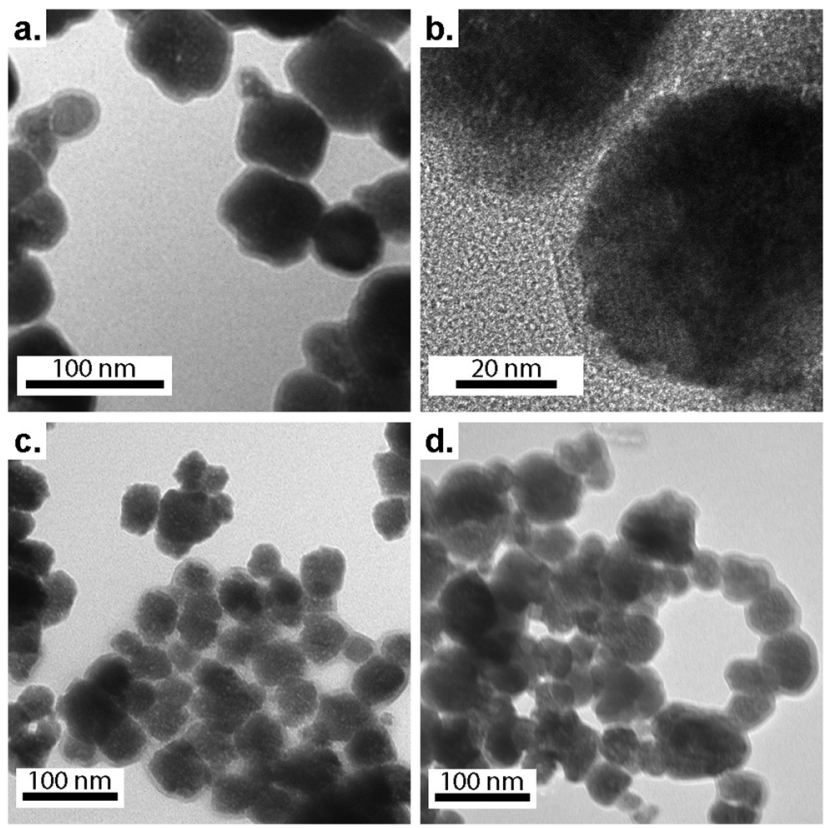

Fig. 2 TEM micrographs of samples BT-1 ( $\mathrm{a}$ and b), BT-3 (c), and BT-5 (d), showing core-shell structures, where the lighter shell is the PEG polymer coating.

be uniform around the NPs in Fig. S6a (ESI $\dagger$ ). The average particle size observed through TEM are in good agreement with data obtained from SEM. All average particle sizes are compiled in Table 1 . The differences between $D_{\mathrm{XRD}}$ and $D_{\mathrm{SEM}}$ or $D_{\mathrm{TEM}}$ are consistent with the presence of a 5-7-nm PEG shell.

According to the literature, a dissolution-precipitation mechanism is responsible for $\mathrm{BaTiO}_{3}$ nanoparticle formation in this work, involving a reaction between $\mathrm{Ti}(\mathrm{OH})_{x}{ }^{4-x}$ hydroxytitanium complexes and barium ions, resulting in the precipitation of $\mathrm{BaTiO}_{3}$ particles. ${ }^{29,41}$ Taking BT-1 as a reference point, a higher concentration of $\mathrm{Ba}^{2+}$ ions in BT-2 decreases the average particle size. This is ascribed to an increase of the nucleation rate; more seeds being present implies the precursor is redistributed on more particles, leading to an average lower size. Similarly, increasing the amount of PEG yields smaller particles. The average particle size with no PEG present (BT-0) was obtained $\approx 110 \mathrm{~nm}$; it decreased to $\approx 60 \mathrm{~nm}$ in BT-1 and further to $\approx 40 \mathrm{~nm}$ for BT-3. We infer the polymer adsorbed on the surface of growing $\mathrm{BaTiO}_{3}$ particles hinders grain growth, resulting in the smaller nanoparticles. A similar behavior has been seen in poly(vinylpyrrolidone)-coated $\mathrm{BaTiO}_{3}$ nanoparticles synthesized by Li et al., ${ }^{29}$ where the particle size decreased upon adding more poly(vinylpyrrolidone). Finally, higher $\mathrm{KOH}$ concentration also accelerates nucleation rate, creating smaller particles (see samples BT-1, BT-4, and BT-5).

\subsection{Nanoparticle coating and water dispersibility}

The presence of the polymer on the nanoparticles' surface was confirmed by ATR-FTIR spectroscopy. Fig. 3 represents the FTIR spectra of PEGylated- $\mathrm{BaTiO}_{3}$ nanoparticles (BT-1, BT-4 and BT-5), as well as neat $\mathrm{PEG}_{400}$. Several peaks are attributed

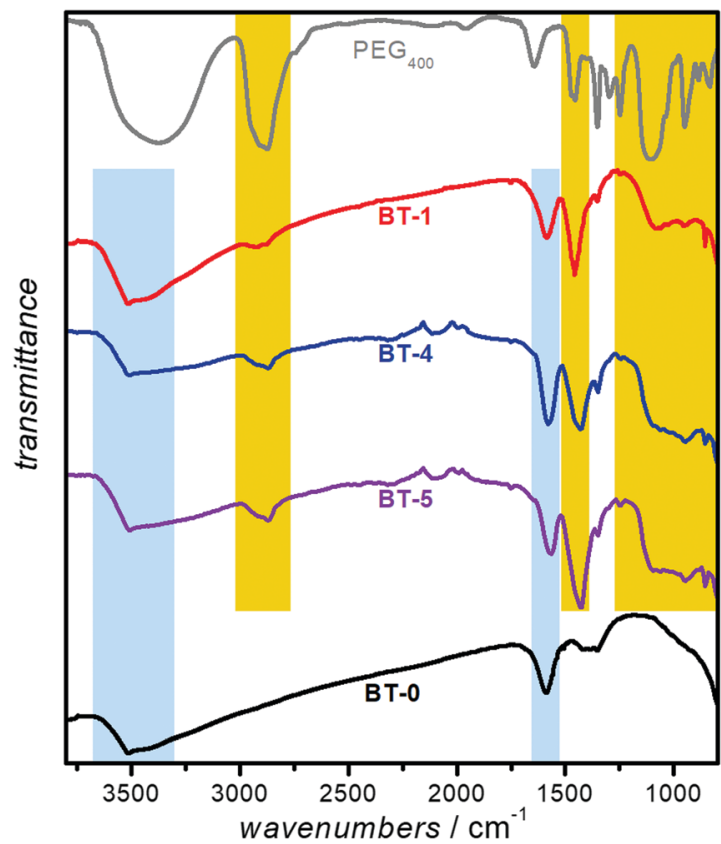

Fig. 3 FT-IR transmittance spectra of (a) BT-0, BT-1, BT-4, BT-5 and $\mathrm{PEG}_{400}$. Common peaks attributed to the PEG coverage are highlighted in yellow and the peaks assigned to $\mathrm{BaTiO}_{3}$ are highlighted in blue.

to the presence of PEG: the C-H out-of-plane bending vibrations (near $950 \mathrm{~cm}^{-1}$ ); in-plane $\mathrm{C}-\mathrm{H}$ and $\mathrm{O}-\mathrm{H}$ as well as $\mathrm{C}-\mathrm{O}-\mathrm{C}$ stretching vibrations (features between 990 to $1250 \mathrm{~cm}^{-1}$ ); $\mathrm{C}-\mathrm{H}$ bending vibrations (near $1450 \mathrm{~cm}^{-1}$ ), and $\mathrm{CH}$ symmetric and asymmetric stretches (bands around 2900 and $2850 \mathrm{~cm}^{-1}$ ), respectively. ${ }^{20}$ As expected, the bands assigned to $\mathrm{PEG}_{400}$ are present in the PEGylated samples (highlighted in yellow), while they are absent in the spectrum of the bare BT-0 nanoparticles. We suggest the PEG is bound to the NP surface through hydrogen bonding between the PEG and surface-terminating hydroxyl groups. $^{22}$ The broad O-H stretching modes of surface-adsorbed water observed around $3500 \mathrm{~cm}^{-1}$ and the deformation mode of absorbed $\mathrm{H}_{2} \mathrm{O}$ molecules, assigned to the bending vibration around $1600 \mathrm{~cm}^{-1}$ were features that have been reported in $\mathrm{BaTiO}_{3}$ FTIR spectrum ${ }^{42}$ which are highlighted in blue in all $\mathrm{BaTiO}_{3}$ samples.

DLS and $\zeta$-potential measurements is a combination of techniques to determine the size distribution of small particles in suspension, and their surface charge. As suspended nanoparticles undergo Brownian motion, they scatter an incident laser beam in all directions; the scattering intensity fluctuates over time due to constructive and destructive interference. An analysis of this scattered light yields the diffusion coefficient of the scattering species; the hydrodynamic diameter is calculated through the Stokes-Einstein equation. The particles aggregation in the dispersion can be recorded by DLS measurements of a sample over time whether the hydrodynamic diameter of the particle increases or not. ${ }^{43,44}$ The $\zeta$-potential, which depends on the surface charge, is a useful parameter to determine the stability of nanoparticles in a suspension. Nanoparticles with $\zeta$-potential magnitude of 20 to $30 \mathrm{mV}$ typically show the highest degrees of stability. ${ }^{44}$ 
a.
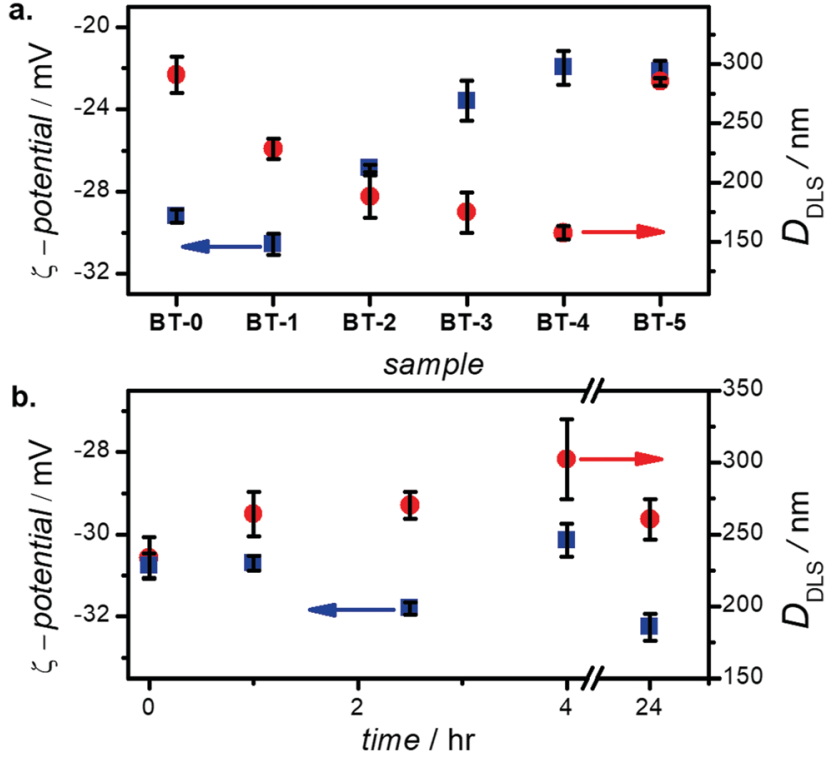

Fig. 4 (a) $\zeta$-potential and hydrodynamic diameter of all $\mathrm{BaTiO}_{3}$ samples, and (b) time variation of $\zeta$-potentials and hydrodynamic diameter of BT-1 nanoparticles over $24 \mathrm{~h}$, indicating stability.

Fig. S7a (ESI $\dagger$ ) illustrates the images of $100 \mathrm{ppm}$ dispersion of $\mathrm{BaTiO}_{3}$ nanoparticles (in DI water, $\mathrm{pH} \approx 7$ ) after $10 \mathrm{~min}$ sonication with an ultrasonic homogenizer $(\approx 4 \mathrm{~kJ}$ energy); Fig. S7b (ESI $\dagger$ ) shows the same dispersions $24 \mathrm{~h}$ later, with no disturbing. This visual test shows that the BT-0 dispersion is more whitish-milky with phase separation, and that the particles clearly precipitate after $24 \mathrm{~h}$ (Fig. S7b, ESI $\dagger$ ), highlighting the importance of the PEG coating for colloidal stability. The large hydrodynamic size ( $\approx 300 \mathrm{~nm}$, see Fig. 4 ) indicates agglomeration and is the likely reason for the low stability of these uncoated nanoparticles in the dispersion.

We examined the nanoparticle's $\zeta$-potential and hydrodynamic diameters ( $D_{\text {DLS }}$ ) (Fig. 4$)$ to better assess their stability in water. All particles show zeta potentials in -31 to $-22 \mathrm{mV}$ range; this is indicative of stable nanoparticles in this medium. ${ }^{44}$ Sample BT-1 shows the highest $\zeta$-potential among all samples $(-30.6 \pm 0.5 \mathrm{mV})$. Their hydrodynamic diameters is around $150-250 \mathrm{~nm}$; the trend generally follows the trends observed in Table 1, although the sizes are larger as they represent the hydrodynamic diameter, not only the inorganic core size. A polymer shell of 10-nm thickness was observed in the TEM images; we however note this is in a dry state. Upon immersion in water, multi-fold swelling of the PEG shell may be expected. ${ }^{45}$ Combined will low-level aggregation (2-3 NPs per agglomerate), this adequately explains the increased hydrodynamic sizes obesevred. Aggregation is likely occurring in sample BT-5, which has a $\zeta$-potential at the limit of colloidal stability, and a larger hydrodynamic diameter.

In order to better understand these nanoparticles' stability, the $\zeta$-potential and hydrodynamic diameter of 100 ppm BT-1 nanoparticles in DI water measured over a 24 h period (Fig. $4 \mathrm{~b}$ ). The measured $\zeta$-potentials show only a slight variation, oscillating around an average value of $-30 \pm 0.5 \mathrm{mV}$, indicating good stability for dispersion of these nanoparticles. Moreover, the negative surface charge of particles due to the electrostatic interaction of surface-modified particles in the aqueous medium might explain the agglomeration delay and enhanced particles' distribution in the solvent. The $D_{\text {DLS }}$ shows a similar behavior albeit with more dispersion - showing an essentially stable diameter of $\sim 225 \mathrm{~nm}$. In general non-ionic PEG polymer acts as a dispersant and prevents agglomeration by creating steric repulsion between particles covered with a hydrated layer in DI water; ${ }^{19}$ therefore, nanoparticles coated with PEG have smaller hydrodynamic particle sizes.

\subsection{Ferroelectric properties}

The relative dielectric constant $\left(\varepsilon_{\mathrm{r}}^{\prime}\right)$ of BT-1 pellet is measured as a function of temperature (Fig. 5); these measurements are conducted on a pellet with a relative density of $86.7 \%$ at a frequency of $10 \mathrm{kHz}$ during heating and cooling cycles. The observed dielectric behaviour is typical of ferroelectric materials, $\varepsilon_{\mathrm{r}}^{\prime}$ increases with temperature to reach the maximum at the ferroelectric-to-paraelectric (FE-PE) transition temperature $\left(T_{\mathrm{C}}\right)$, and then decreases. The observed $T_{\mathrm{C}}$ of $\approx 130{ }^{\circ} \mathrm{C}$ is in close agreement with the reported phase transition for $\mathrm{BaTiO}_{3}$ ceramics. ${ }^{1}$ The $\varepsilon_{\mathrm{r}}^{\prime}$ value of $\approx 2900$ at room temperature and
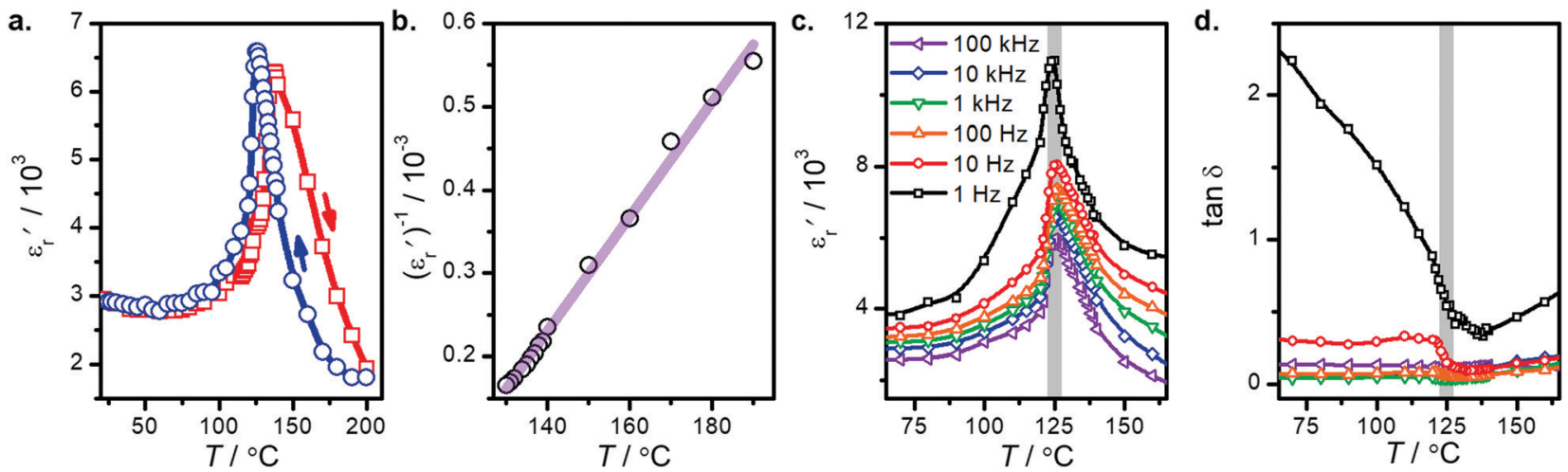

Fig. 5 (a) Temperature dependence of the dielectric constant $\left(\varepsilon_{\mathrm{r}}^{\prime}\right)$ for BT-1 at a frequency of $10 \mathrm{kHz}$ showing heating $\left.(\square)\right)$ and cooling $\left({ }^{\circ}\right) \mathrm{cycles}$. (b) Data fit to the Curie-Weiss law. Variation of (c) dielectric constant $\varepsilon_{\mathrm{r}}^{\prime}$ and (d) loss tangent $(\tan \delta)$ with temperature at different frequencies for sample BT-1. 
$\varepsilon_{\mathrm{r}}^{\prime}(\max )$ of 6500 at the $T_{\mathrm{C}}$ indicate that BT-1 is a strong ferroelectric compound, owing to its tetragonal structure. Above this transition temperature (paraelectric region), $\varepsilon_{\mathrm{r}}^{\prime}$ follows the CurieWeiss law,

$$
\varepsilon_{\mathrm{r}}^{\prime}=\frac{A}{T-T_{\mathrm{C}}}
$$

where $A$ is a constant (determined from the slope of $1 / \varepsilon_{\mathrm{r}}^{\prime}$ versus $\left(T-T_{\mathrm{C}}\right.$ ) (see Fig. 5b). The determined Curie-Weiss constant of $A \approx 14.7 \times 10^{4}$ is in the same range of reported values in literature. ${ }^{3}$ The obtained heating and cooling cycles show a thermal hysteresis at $T_{\mathrm{C}}$ (difference between $T_{\mathrm{C}}$ (heating) and $T_{\mathrm{C}}$ (cooling)), indicating that the ferroelectric-paraelectric anomaly is first-order. ${ }^{46,47}$ The transition temperature detected on heating $\left(T_{\mathrm{C}}=135{ }^{\circ} \mathrm{C}\right)$ is usually larger than the transition temperature observed on cooling $\left(T_{\mathrm{C}}=125{ }^{\circ} \mathrm{C}\right)$. The thermal hysteresis of $10{ }^{\circ} \mathrm{C}$ at the $\mathrm{FE}-\mathrm{PE}$ transition temperature of $\mathrm{BaTiO}_{3}$ has already been reported by Baskaran et al., ${ }^{46}$ although the hysteresis is often reported to be less than $5{ }^{\circ} \mathrm{C}$. ${ }^{3,47}$

The temperature dependence of $\varepsilon_{\mathrm{r}}^{\prime}$ and loss tangent $(\tan \delta)$ during cooling cycle, at different frequencies, are shown in Fig. $5 \mathrm{c}-\mathrm{d}$. We observe that $\varepsilon_{\mathrm{r}}^{\prime}$ and $\tan \delta$ decrease, and that the Curie-Weiss transition temperature slightly shifts to a lower temperature with increasing frequency. The highest value of $\varepsilon_{\mathrm{r}}^{\prime}$ at $1 \mathrm{~Hz}$ is 11000 at $T_{\mathrm{C}}=126{ }^{\circ} \mathrm{C}$. The lowest value of 5950 at the transition temperature is also recorded for the frequency of $100 \mathrm{kHz}$.

When preparing pellets, there is always a concern as to how the pellet sintering and pressing affects the crystal sizes, and hence the resultant properties. We attempted to minimize such effects by doing a two-step sintering process previously shown to yield high-density pellets, with minimal effect on the NP size. ${ }^{33}$ In order to understand the effect of heating on the crystal structure of particles, the lattice parameters and crystallite size of ground pellet of BT-1 were obtained via PXRD analysis (Fig. S8, ESI $\dagger$ ). Results show that crystallite sizes slightly increased from $39.8 \pm 2.1 \mathrm{~nm}$ to $42.6 \pm 3.9 \mathrm{~nm}$; in doing so, the tetragonality was enhanced to $c / a=1.008$. According to Wada et al., large values of dielectric constant in nanoparticles can be attributed to the existence of uniform grains, easier domain walls movement, surface effect and also higher local space charge field due to the large surface area. ${ }^{48}$ In our case, uniform grains and enhanced tetragonality due to the two-step sintering technique creates this large FE response.

\section{Conclusions}

In summary, easily water-dispersed $\mathrm{BaTiO}_{3}$-PEG core-shell particles with an average particle size of $60 \mathrm{~nm}$ and a tetragonality value of 1.005 were prepared using a simple and fast low-temperature synthesis method. The impact of experimental conditions on particle size and crystal structure were studied, confirming lattice expansion and tetragonality reduction in smaller particles. Surface bonding between $\mathrm{BaTiO}_{3}$ particles and PEG molecules were studied using FT-IR. The measured $\zeta$-potential of $\approx-30 \mathrm{mV}$ is indicative of good stability and redispersibility of these surface-modified particles. Furthermore, the large values of dielectric constant $(\approx 6000-11000)$ at the transition temperature of $T_{\mathrm{C}}=125-135{ }^{\circ} \mathrm{C}$ confirm the strong ferroelectricity in this sample. These findings provide a simple solution to nanoparticle aggregation, thereby improving the stability of ferroelectric dispersion.

\section{Conflicts of interest}

There are no conflicts to declare.

\section{Acknowledgements}

We thank the Natural Sciences and Engineering Research Council of Canada (NSERC; Discovery Grant, RTI, and PDF), the Canada Excellence Research Chair (CERC) program, and the University of Calgary's Global Research Initiative in Sustainable Low Carbon Unconventional Resources funded by the Canada First Research Excellence Fund (CFREF) for supporting this research. We thank Dr Berton for performing thermal analysis and 4D LABS (Simon Fraser University) for HRTEM imaging.

\section{Notes and references}

1 M. Acosta, N. Novak, V. Rojas, S. Patel, R. Vaish, J. Koruza, G. A. Rossetti and J. Rodel, Appl. Phys. Rev., 2017, 4, 041305.

2 B. Jiang, J. Iocozzia, L. Zhao, H. Zhang, Y. Harn, Y. Chen and Z. Lin, Chem. Soc. Rev., 2019, 48, 1194-1228.

3 Z. Zhao, V. Buscaglia, M. Viviani, M. T. Buscaglia, L. Mitoseriu, A. Testino, M. Nygren, M. Johnsson and P. Nanni, Phys. Rev. B: Condens. Matter Mater. Phys., 2004, 70, 024107.

4 C. Xiao, Z. Chi, W. Zhang, F. Li, S. Feng, C. Q. Jin, X. Wang, X. Deng and L. Li, J. Phys. Chem. Solids, 2007, 68, 311-314.

5 K. Uchino, E. Sadanaga and T. Hirose, J. Am. Ceram. Soc., 1989, 72, 1555.

6 Z. G. Shen, J. F. Chen, H. K. Zou and J. Yun, J. Colloid Interface Sci., 2004, 275, 158-164.

7 A. Hirata, Y. Nitta and M. Kawabata, J. Ceram. Soc. Jpn., 1994, 102, 1168-1172.

8 G. Ciofani, S. Danti, S. Moscato, L. Albertazzi, D. D’Alessandro, D. Dinucci, F. Chiellini, A. Petrini and M. Menciassi, Colloids Surf., B, 2010, 76, 535-543.

9 J. Li, K. Inukai, A. Tsuruta, Y. Takahashi and W. Shin, J. Asian. Ceram. Soc., 2017, 5, 444-451.

10 C. Hai, K. Inukai, Y. Takahashi, N. Izu, T. Akamatsu, T. Itoh and W. Shin, Mater. Res. Bull., 2014, 57, 103-109.

11 X. Pang, Y. He, B. Jiang, J. Iocozzia, L. Zhao, H. Guo, J. Liu, M. Akinc, N. Bowler, X. Tan and Z. Lin, Nanoscale, 2013, 5, 8695-8702.

12 B. Jiang, X. Pang, B. Li and Z. Lin, J. Am. Chem. Soc., 2015, 137, 11760-11767.

13 X. Wang, T. Karaki, K. Koyanagi and T. Fujii, Jpn. J. Appl. Phys., 2020, 59, SCCB05.

14 L. S. Simon-Seveyrat, A. Hajjaji, Y. Emziane, B. Guiffard and D. Guyomar, Ceram. Int., 2007, 33, 35-40. 
15 G. Panomsuwan and H. Manuspiya, J. Appl. Phys. A, 2018, 124(10), 1-8.

16 S. K. Lee, T. J. Park, G. J. Choi, K. K. Koo and S. W. Kim, Mater. Chem. Phys., 2003, 82, 742-749.

17 S. G. Kwon, B. H. Park, K. Choi, E. S. Choi, S. Nam, J. W. Kim and J. H. Kim, J. Eur. Ceram. Soc., 2006, 26, 1401-1404.

18 S. S. Tripathy and A. M. Raichur, J. Exp. Nanosci., 2011, 6, 127-137.

19 J. S. Suk, Q. Xu, N. Kim, J. Hanes and L. M. Ensign, Adv. Drug Delivery Rev., 2016, 99, 28-51.

20 A. Banerjee, B. Blasiak, E. Pasquier, B. Tomanek and S. Trudel, RSC Adv., 2017, 7, 38125.

21 J. S. Suk, Q. Xu, N. Kim, J. Hanes and L. M. Ensign, Adv. Drug Delivery Rev., 2016, 99, 28-51.

22 A. S. Karakoti, S. Das, S. Thevuthasan and S. Seal, Angew. Chem., Int. Ed., 2011, 50, 1980-1994.

23 S. Moharana, M. K. Mishra, B. Behera and R. N. Mahalinga, Polym. Sci., 2017, 59, 405-415.

24 J. G. Kim, W. P. Tai, K. J. Lee and W. S. Cho, Ceram. Int., 2004, 30, 2223-2227.

25 J. Čulić-Viskota, W. P. Dempsey, S. E. Fraser and P. Pantazis, Nat. Protoc., 2012, 7(9), 1618-1633.

26 J. Zhu, W. Li, X. Huo and Y. Zhu, J. Phys. D: Appl. Phys., 2015, 48, 355301.

27 R. B. Upadhyay, S. Annam, M. R. Patel, A. K. Sharma, P. Mevada and U. S. Joshi, Ferroelectr., Lett. Sect., 2016, 43, 25-33.

28 S. W. Boland, PhD thesis, California Institute of Technology, 2005.

29 J. Li, K. Inukai, Y. Takahashi and W. Shin, J. Asian. Ceram. Soc., 2016, 4, 394-402.

30 T. Roisnel and J. Rodriguez-Carvajal, in Proceedings of the Seventh European Powder Diffraction Conference, ed. R. Delhez and E.J. Mittenmeijer, 2000, pp. 118-123.

31 D. M. Smilgies, J. Appl. Crystallogr., 2009, 42, 1030-1034.

32 C. A. Schneider, W. S. Rasband and K. W. Eliceiri, Nat. Methods, 2012, 9, 671-675.
33 M. Taheri, B. Zanca, S. J. Villegas, M. Dolgos, S. Bryant and S. Trudel, 2020 Joint Conference of the IEEE International Frequency Control Symposium and International Symposium on Applications of Ferroelectrics (IFCS-ISAF), 2020, pp. 1-4.

34 Y. Gao, V. V. Shvartsman, A. Elsukova and D. C. Lupascu, J. Mater. Chem., 2012, 22, 17573-17583.

35 S. Yoon, S. Baikw, M. G. Kim, N. Shin and I. Kim, Am. Ceram. Soc., 2007, 90, 311-314.

36 O. G. Grendal, A. B. Blichfeld, S. L. Skjærvø, W. V. Beek, S. M. Selbach, T. Grande and M. A. Einarsrud, Crystals, 2018, 8, 253.

37 M. B. Smith, K. Page, T. Siegrist, P. L. Redmond, E. C. Walter, R. Seshadri, L. E. Brus and M. L. Steigerwald, J. Am. Chem. Soc., 2008, 130, 6955-6963.

38 P. M. Diehm, P. Agoston and K. Albe, Chem. Phys. Chem., 2012, 13, 2443-2454.

39 S. Tsunekawa, K. Ishikawa, Z. Q. Li, Y. Kawazoe and A. Kasuya, Phys. Rev. Lett., 2000, 85, 3440.

40 H. Hayashi, T. Nakamura and T. Ebina, J. Phys. Chem. Solids, 2013, 74(7), 957-962.

41 H. Xu and L. Gao, Mater. Lett., 2002, 57, 490-494.

42 A. Aala, T. R. Hammad, M. Zawrah, I. K. Battisha and A. B. Abou Hammad, Acta Phys. Pol., A, 2014, 126, 1318-1321.

43 J. Stetefeld, S. McKenna and T. Patel, Biophys. Rev., 2016, 8, 409-427.

44 S. Bhattacharjee, J. Controlled Release, 2016, 235, 337-351.

45 A. V. Salvekar, W. M. Huang, R. Xiao, Y. S. Wong, S. S. Venkatraman, K. H. Tay and Z. X. Shen, Acc. Chem. Res., 2017, 50, 141-150.

46 N. Baskaran, A. Ghule, C. Bhongale, R. Murugan and H. Chang, J. Appl. Phys., 2002, 91, 10038.

47 D. Ricinschi, V. Tura, L. Mitoseriu and M. Okuyama, J. Phys.: Condens. Matter, 1999, 11, 1601-1613.

48 S. Wada, T. Hoshina, K. Takizawa, M. Ohishi, H. Yasuno, H. Kakemoto, T. Tsurumi, C. Moriyoshi and Y. Kuroiwa, J. Korean Phys. Soc., 2007, 51(2), 878-881. 